\title{
Surgical therapy and next-generation sequencing- based genetic alteration analysis of malignant solitary fibrous tumor of the pleura
}

This article was published in the following Dove Press journal:

OncoTargets and Therapy

\author{
Zuoqing Songl,* \\ Fan Yangl,* \\ Yingguo Zhang ${ }^{2}$ \\ Ping Fan ${ }^{2}$ \\ Guowei Liu ${ }^{2}$ \\ Chao $\mathrm{Li}^{2}$ \\ Wansheng Ding ${ }^{2}$ \\ Yulong Zhang ${ }^{2}$ \\ Xiaohong $\mathrm{Xu}^{3}$ \\ Yancheng $\mathrm{Ye}^{2}$ \\ 'Department of Lung Cancer Surgery, \\ Tianjin Medical University General \\ Hospital, Tianjin 300052, China; \\ ${ }^{2}$ Wuwei Tumour Hospital, Gansu \\ Province 733000 , China; ${ }^{3}$ College of \\ Nursing, Tianjin Medical University, \\ Tianjin 300070, China \\ *These authors contributed equally \\ to this work
}

Correspondence: Yancheng Ye Wuwei Tumour Hospital, No. 3I, Weishengxiang, Haizang Road, Wuwei 733000, China

Email yancye@।39.com

Xiaohong Xu

College of Nursing, Tianjin Medical University, No. 22 Qixiangtai Road, Heping District, Tianjin 300070, China Emailmiao1980@aliyun.com
Background: Solitary fibrous tumor of the pleura (SFTP) is a rare pleural neoplasm arising from mesenchymal cells, accounting for $<5 \%$ of pleural neoplasms. Approximately $10 \%$ of cases of SFTP demonstrate malignant potential, leading to local recurrence after radical surgery and subsequent metastasis.

Methods: A large malignant-like mass was found in the left thoracic cavity of a 61-year-old woman. Following radical resection of the mass, the patient was diagnosed with malignant SFTP by histologic and immunohistochemical analyses. In addition, a next-generation sequencingbased mutation test was used to reveal the mutational profile of the tumor. The genetic alteration panel was analyzed with reference to public data on the ClinVar and COSMIC databases, after which the public SFTP data were analyzed for frequency of altered genes. Finally, through overlay of the abovementioned two sets, the genetic alteration accounting for SFTP initiation was anticipated to be identified.

Results: In the mutation panel of our malignant SFTP group, kinase insert domain receptor (KDR) and fms-related tyrosine kinase 1 (FLT1) scored high in pathogenesis but had only a medium frequency; the NAB2-STAT6 fusion appeared to be the dominant genetic alteration in public SFTP samples.

Conclusion: The high frequency of NAB2-STAT6 fusion indicates its prominent role in SFTP, while somatic mutations such as FLT1-R593W and KDR-V297I may also contribute to the malignant angiogenic phenotype. The present study affirmed the heterogeneity of SFTP, and more sophisticated classification methods will be needed to explore its underlying mechanisms.

Summary: We believe that improvement in the prognosis of SFTP relies on early diagnosis, margin-free resection, and long-term follow-up. Through genetic analysis, it appears that both NAB2-STAT6 fusion and somatic mutations such as FLT1-R593W and KDR-V297I contribute to SFTP development.

Keywords: malignant solitary fibrous tumor of the pleura, surgery, pleura, mediastinum, next-generation sequencing

\section{Introduction}

Solitary fibrous tumors (SFTs) represent a group of neoplasms with fibroblastic mesenchymal origin that can be found in almost any anatomic site. ${ }^{1}$ Since the first report of SFT of the pleura (SFTP) in $1870,{ }^{2}$ the pleurae have been considered the most common site of SFT, ${ }^{3,4}$ while this type of tumor has also been found in other sites, including meninges, soft tissues, bone, dermis, gastrointestinal tract, ${ }^{5}$ spinal cord, ${ }^{6}$ bladder wall, blood vessels, ${ }^{7}$ and heart. ${ }^{4}$ Histologically, SFTs are characterized by variable and irregular distribution of bland spindle and oval cells, as well 
as prominent collagenous stroma and diffuse expression of CD34. ${ }^{1}$ SFTP accounts for $5 \%$ of all tumors of the pleura, and is mainly benign. Malignant solitary fibrous tumor of the pleura (MSFTP) is relatively rare and constitutes $\sim 20 \%$ of all reported SFTPs. ${ }^{8}$ In this report we present the case of a female patient with a malignant pleural tumor invading adjacent mediastinal structures. The patient underwent en bloc resection with wide margins of adjacent tissue. The tumor was subsequently confirmed to be an MSFTP by pathologic diagnosis. In this study, we analyzed the genetic characteristics of one case of MSFTP and utilized the public somatic mutation database to discover potential genetic alterations in common, which could act as initiating or driving factors in tumor progression. We also discuss mutations that could explain certain phenotypes of cases described here.

\section{Methods \\ Diagnosis and surgical treatment}

A 61-year-old woman was hospitalized with complaints of chronic left flank pain, moderate dyspnea, and a chronic cough that had persisted for about 2 months. Her past medical history was unremarkable. Enhanced chest computed tomography (CT) revealed an $\sim 16 \times 13 \times 11 \mathrm{~cm}^{3}$ mass in the left thoracic cavity and the left upper abdominal cavity, directly invading the mediastinum and compressing multiple adjacent organs including the pericardium, diaphragm, aorta tunica vaginalis, the left upper lobe lingular segment of the lung, part of the left lower lobe of the lung, and the left lobe of the liver (Figure 1A-C). Contrast agent enhancement of blood vessels revealed pathologic branching of vessels, originating from arteries such as the descending aorta (white arrows in Figure 1D), nourishing the mass in a deregulated
A

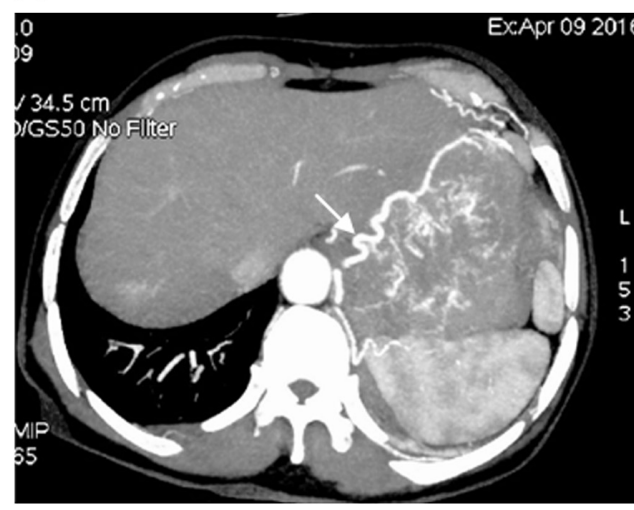

C

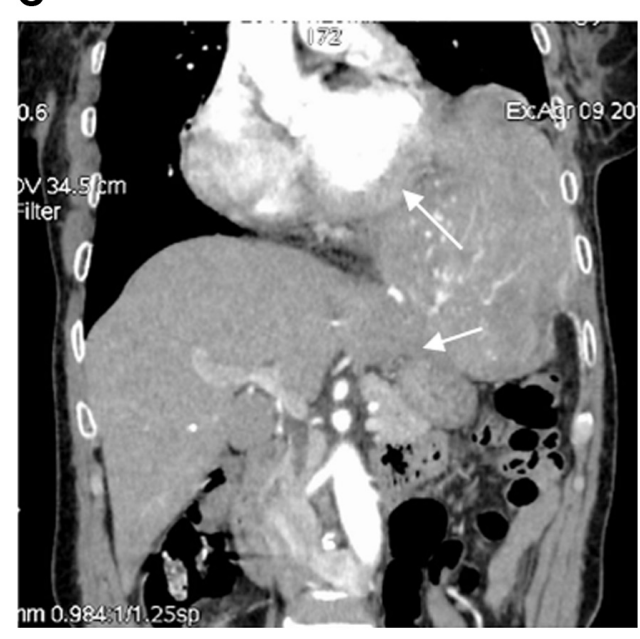

B

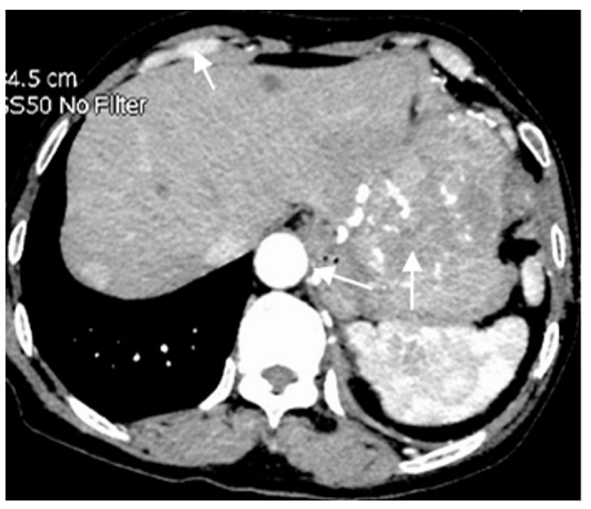

D

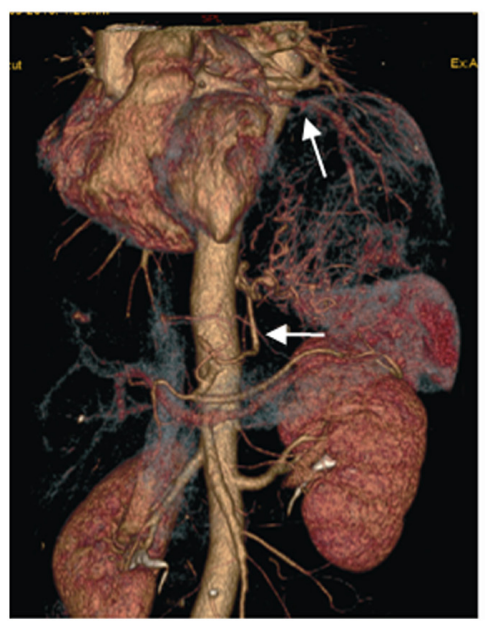

Figure I Enhanced chest CT scan, reconstructed coronal CT image, and reconstructed angiography of chest CT.

Notes: (A) Giant and invasive malignant solitary fibrous tumor of the pleura in the left hemithorax and abdominal cavity, with serpiginous vessels. (B) Inhomogeneous enhancement and heterogeneous map-like pattern. (C) Tumor invasion of pericardium (upper white arrow), diaphragm, and left lobe of liver (lower white arrow). (D) Two arterial vessels (arrows) nourishing tumor.

Abbreviation: CT, computed tomography. 
manner (Figure 1A). In addition, the mass demonstrated a heterogeneous enhancement, an indication of tumor malignancy. A number of nodules were also found in the thoracic cavity. Physical examination revealed decreased sounds on auscultation and dull notes over the left lower hemithorax. Preoperative laboratory evaluation, electrocardiography, transthoracic echocardiogram, and radiologic examinations of other sites revealed no significant abnormalities.

The patient underwent radical resection of the thoracic tumor by left posterolateral thoracotomy. This procedure was extremely difficult, including not only resection of the giant mass occupying the thoracic cavity and mediastinum, invaded left upper lobe lingular segments of the lung, partial left lower lobe of the lung, multiple pleural metastatic nodules, partial aortic tunica vaginalis, and pericardium, but also resection of the invaded diaphragm and subsequent reconstruction, as shown in Figure 2A and B. Specimens of the tumor mass were used for the pathologic diagnosis and genetic analysis. Ten days after the operation, the patient recovered well and was discharged.

\section{Immunohistochemical analysis}

Formalin-fixed, paraffin-embedded (FFPE) blocks of tumor tissue were sliced into $5 \mu \mathrm{m}$ sections, deparaffinized in xylene $(3 \times 5$ minutes $)$ and then rehydrated through graded concentrations of ethanol $(2 \times 2$ minutes in $100 \%$ ethanol, $1 \times 2$ minute in $95 \%$ ethanol, $1 \times 2$ minute in $75 \%$ ethanol). Tissue sections were then heated in EDTA solution (ZLI-9067; ZSGB-BIO, Beijing, China), for 150 seconds. After cooling, the sections were washed with PBS and then incubated with primary antibodies at $4^{\circ} \mathrm{C}$ overnight. The primary antibodies used were rabbit antibodies against CD34 (ZA-0550; ZSGBBIO), CD99 (ZA-0577; ZSGB-BIO), Bcl-2 (ZA-0536;
ZSGB-BIO), Ki67 (ZA-0502; ZSGB-BIO), and S100 (ZA-0225; ZSGB-BIO), as well as mouse antibodies against CK5/6 (ZM-0313; ZSGB-BIO) and CK-pan (ZM-0069; ZSGB-BIO). Slides were washed again and incubated with secondary antibodies using a PV-9000 kit (ZSGB-BIO) according to the manufacturer's manual. The slides were then stained with hematoxylin (Absin, Fuzhou, China).

\section{Next-generation sequencing (NGS) analysis}

The FFPE tissue sections were sent to SurExam ${ }^{\text {TM }}$ Biotech Co. Ltd. (Shenzhen, China) for NGS. A commercialized mutation analysis for a panel of 398 genes was used to detect alterations in 398 tumor-correlated genes. The slided $5 \mu \mathrm{m}$ thick PPFE sample was collected and purified by using the Qiamp DNA Mini kit (Qiagen ${ }^{\mathrm{TM}}$, Hilden, Germany) according to the protocol described in the manufacturer's instruction. Next, the DNA sample was quantified by nanodrop 1000

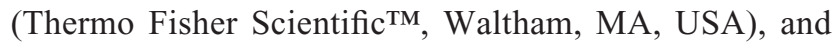
stored at $-20^{\circ} \mathrm{C}$ until used. The DNA library was prepared by using Ion AmpliSeq Library Kit 2.0 (Thermo Fisher Scientific $^{\mathrm{TM}}$, USA) on Tecan Freedom EVO platform (Tecan ${ }^{\mathrm{TM}}$, Männedorf, Switzerland), quantified by the Qubit 2.0 Fluorometer (Thermo Fisher Scientific ${ }^{\mathrm{TM}}$, USA), prepared on Ion Chef system (Thermo Fisher Scientific ${ }^{\mathrm{TM}}$, USA), and finally sequenced on Ion Proton ${ }^{\mathrm{TM}}$ Sequencer (Thermo Fisher Scientific, USA). The Torrent Suite ${ }^{\text {TM }}$ (Thermo Fisher Scientific, USA) was used to process signal and data qualification, the Torrent Mapper ${ }^{\mathrm{TM}}$ (Thermo Fisher Scientific ${ }^{\mathrm{TM}}$, USA) was used to reference with the GRCh 37/hg 19 database, and the Torrent Variant Caller software (Thermo Fisher Scientific ${ }^{\mathrm{TM}}$, USA) was used for mutation calling. Afterward, the genetic alterations were confirmed by amplifying the genes with specific primers (Table 1) and by Sanger sequencing. PCR
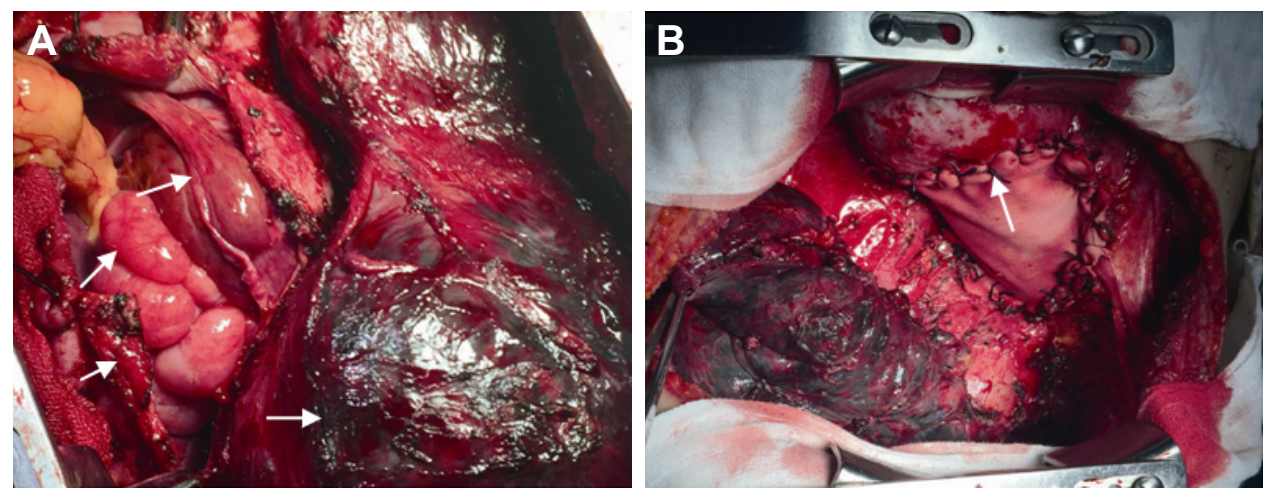

Figure 2 Intraoperative photographs showing the mass of the invasive malignant solitary fibrous tumor of the pleura and reconstructed diagram.

Notes: Radical resection of thoracic tumor through left posterolateral thoracotomy. (A) Tumor-invaded diaphragm, upper lobe of left lung, and left lobe of liver, with a number of nodules in the abdominal cavity. (B) Radical resection of thoracic tumor and reconstruction of diaphragm with pericardial polyester patch. The arrows indicate tumor tissue. 
Table I The primers used in Sanger sequencing

\begin{tabular}{|c|c|c|c|c|}
\hline Gene & Primer name & Primer sequence & $\begin{array}{l}\text { Annealing } \\
\text { temperature }\end{array}$ & $\begin{array}{l}\text { Area } \\
\text { covered }\end{array}$ \\
\hline \multirow[t]{2}{*}{ ARIDIA } & ARIDIA-chrI-F & GTTTAGGACGGAGCCTGGAA & 59.3 & 262 \\
\hline & ARIDIA-chrI-R & TTGGGTGGAGAACTGATTGC & 58.1 & \\
\hline \multirow[t]{2}{*}{ PTGS2 } & PTGS2-chrl-F & GTATGACTCCTTTCTCCGCAA & 57.1 & 333 \\
\hline & PTGS2-chrl-R & CAAATCATCAACACTGCCTCAA & 58.6 & \\
\hline \multirow[t]{2}{*}{ ALK } & ALK-chr2-FI & CTCCTTCATATTGGCAGTCAGC & 59.2 & 241 \\
\hline & ALK-chr2-RI & GTTGCACAAGGTCCACGGA & 59.5 & \\
\hline \multirow[t]{2}{*}{ ALK } & ALK-chr2-F2 & TTACCCCTGTCGTGTGGCT & 58.3 & 267 \\
\hline & ALK-chr2-R2 & TCTGCCTACCACСTCСTCTG & 57.3 & \\
\hline \multirow[t]{2}{*}{ SETD2 } & SETD2-chr3-FI & ATCTGGCTGTTCTTGGCTCCT & 60 & 296 \\
\hline & SETD2-chr3-RI & GCAAGGATGGCAAAGAGGAT & 59 & \\
\hline \multirow[t]{2}{*}{ SETD2 } & SETD2-chr3-F2 & TCATACAAATGTCTCCTTGACTCCA & 60 & 268 \\
\hline & SETD2-chr3-R2 & GTCATGCCCCAGAAATTGTGT & 59.6 & \\
\hline \multirow[t]{2}{*}{ MAGII } & MAGII-chr3-F & CAGACCAATGAGAACATCAAACAA & 59.5 & 286 \\
\hline & MAGII-chr3-R & CTTTTAACATAACAGCCATGACCTT & 59.4 & \\
\hline \multirow[t]{2}{*}{ GATA2 } & GATA2-chr3-F & AGTGACACCTGGTACTTGACGC & 59 & 292 \\
\hline & GATA2-chr3-R & ССTTCTCCAAGACGCCACT & 57.3 & \\
\hline \multirow[t]{2}{*}{ TNK2 } & TNK2-chr3-F & ACCACGCCAAAGGAACCAT & 59.9 & 296 \\
\hline & TNK2-chr3-R & ACCTATCTGGAAGCCGAAGC & 58.6 & \\
\hline \multirow[t]{2}{*}{ SYNEI } & SYNEI-chr6-F & TTCACACATTTTCACTGACGCT & 58.3 & 255 \\
\hline & SYNEI-chr6-R & AAAGCAGTTCTCATAATCTCAGCAT & 59.1 & \\
\hline \multirow[t]{2}{*}{ EGFR } & EGFR-chr7-F & GGGTCCCTGCTCTGTCACTG & 59.9 & 277 \\
\hline & EGFR-chr7-R & GAAGAGAAAATCAAACAGAATGCC & 59.5 & \\
\hline \multirow[t]{2}{*}{ AKAPQ } & AKAP9-chr7-F & TGAATCATAAGTAGAACCAGGGC & 58.2 & 305 \\
\hline & AKAP9-chr7-R & GAATTGGTGATCCATTGCTCC & 59.2 & \\
\hline \multirow[t]{2}{*}{ CDKN2A } & CDKN2A-chr9-F & TGGTTACTGCCTCTGGTGCC & 60.7 & 309 \\
\hline & CDKN2A-chr9-R & ACCCTGGCTCTGACCATTCT & 58.3 & \\
\hline \multirow[t]{2}{*}{ PAX5 } & PAX5-chr9-F & CССTCAATAGGTGCCATCAGT & 59 & 264 \\
\hline & PAX5-chr9-R & GGGAACAGACTGGGACAGAGAT & 59.5 & \\
\hline \multirow[t]{2}{*}{ PTCHI } & PTCHI-chr9-F & GCTGGGTTCCGAGGGTTG & 60.7 & 241 \\
\hline & PTCHI-chr9-R & AATCCAGGCATCACCCACC & 59.9 & \\
\hline \multirow[t]{2}{*}{$\mathrm{TSCl}$} & TSCI-chr9-F & AGTGGTGGGGATGCTGGC & 60.9 & 219 \\
\hline & TSCI-chr9-R & GTAGGGCACAATGAAGAGGC & 57.1 & \\
\hline \multirow[t]{2}{*}{$\mathrm{NOTCHI}$} & NOTCHI-chr9-F & TCCTGGGGCAGAATAGTGTG & 58.1 & 306 \\
\hline & NOTCHI-chr9-R & CCTGTCCCAGATGATGAGCTA & 57.4 & \\
\hline \multirow[t]{2}{*}{ RET } & RET-chrI0-F & TTCTCСTTCATCGTCTCGGTG & 60 & 313 \\
\hline & RET-chrl0-R & GTTGGCAGCCCCTCACAG & 59 & \\
\hline TETI & TETI-chrl0-F & AATTGTGCATTAACCACCATCAG & 59.4 & 251 \\
\hline & TETI-chrI0-R & CAAAGCAAGACCCCACCTCA & 60 & \\
\hline MENI & MENI-chrlI-F & GGTGGACACTTTCTGCTTCTTC & 58 & 294 \\
\hline & MENI-chrlI-R & AAGCCAGCACTGGACAAGG & 58 & \\
\hline HNFIA & HNFIA-chrI2-F & CAAGGAGTTTGGTTTGTGTCTG & 57.4 & 366 \\
\hline & HNFIA-chrI2-R & AGGATGGGTGGCGTGAAG & 58.5 & \\
\hline LAMPI & LAMPI-chrl3-F & ATAAATGTGTGAATCTACTGGGGTT & 58.5 & 253 \\
\hline & LAMPI-chrI3-R & GCAGCTTTAAAGGCAGGGT & 57.3 & \\
\hline$B C L I \mid B$ & BCLIIB-chrI4-F & CGGTTGCGGCTCAGCTC & 60 & 272 \\
\hline & BCLIIB-chr|4-R & ACGCACATGCACAAGGCC & 60 & \\
\hline TSC2 & TSC2-chrl6-F & GAGCCAAGTCTGTTCCGTTC & 57 & 299 \\
\hline & TSC2-chrl6-R & GGAGGGAGGCCCAGCAG & 60 & \\
\hline $\mathrm{CDHI}$ & $\mathrm{CDHI}-\mathrm{chrl6}-\mathrm{F}$ & TCTGGTGGAAGGCAATGG & 56.8 & 294 \\
\hline & $\mathrm{CDHI}-\mathrm{chrl6-R}$ & TTGGGTCGTTGTACTGAATGGT & 59.5 & \\
\hline TP53 & TP53-chrl7-F & AGAATGCAAGAAGCCCAGAC & 56.8 & 264 \\
\hline & TP53-chrl7-R & ACCCATCTACAGTCCCCCTT & 57.3 & \\
\hline WAS & WAS-chrX-F & CAGAAGAAATCAATGAGAGTTACAGC & 59.2 & 258 \\
\hline & WAS-chrX-R & TGGTCCAGAACGTCCAGTAGC & 59.9 & \\
\hline ATRX & ATRX-chrX-F & AGTTTCATCGCTCTGGTCTTTCT & 59 & 258 \\
\hline & ATRX-chrX-R & CAGAAGGCACAGTTGATAAAGACA & 59 & \\
\hline
\end{tabular}

Abbreviations: ALK, anaplastic lymphoma receptor tyrosine kinase; ATRX, alpha thalassemia/mental retardation syndrome $X$-linked; EGFR, epidermal growth factor receptor; MENI, menin I; PAX5, paired box 5; RET, ret proto-oncogene; SETD2, SET domain containing 2; SYNEI, spectrin repeat containing nuclear envelope protein I; TP53, tumor protein p53. 
was performed on a Veriti ${ }^{\mathrm{TM}}$ 96-well Thermal Cycler (Thermo Fisher Scientific, USA) and the products were purified (SK1141; Sangon Biotech ${ }^{\mathrm{TM}}$, Shanghai, China) using agarose gel electrophoresis (DYY-8C; Beijing Liuyi ${ }^{\mathrm{TM}}$ Biotechnology Co. Ltd., Beijing, China) and then sequenced with a 3730XL DNA Analyzer (Thermo Fisher Scientific ${ }^{\mathrm{TM}}$, USA).

Subsequently, the genetic alterations reconfirmed by Sanger sequencing were searched in the ClinVar database (http://www.ncbi.nlm.nih.gov/clinvar/) for single-nucleotide polymorphism code, clinical significance, and called variants, which are the samples submitted to $\mathrm{dbGaP}$ that possess the variant allele. The mutations were also searched in the PolyPhen-2 database (http://genetics.bwh.harvard.edu/pph2/) and the Catalog of Somatic Mutations in Cancer (COSMIC, http://cancer.sanger.ac.uk/cosmic) database to obtain the predicted influence of the mutated gene upon the original protein function, which is quantified in both databases as the PolyPhen-2 score and FATHMM score. Higher scores indicate greater possibility of damaging the effect on the physiologic function. The mutated genes were then categorized based on whether they had high PolyPhen-2 or FATHMM scores or had a frequency of greater than $90 \%$. In addition, all sequencing data for SFTs on COSMIC were summarized and analyzed for the frequencies of all altered genes. Finally, two groups of genes, the validated altered genes in the present case and reported altered genes, were overlapped to identify a shared altered gene set.

\section{Ethics statement}

The experiment was performed in accordance with relevant guidelines and regulations and approved by Wuwei Tumour Hospital Ethics Committee. Written informed consent was obtained from the patient for the treatment and for publication of the case details.

\section{Results}

Diagnosis of SFTP was finally made by pathology. The preoperative radiographic tests revealed the invasion of the mediastinum, richness of pathologic nourishing vessels as well as the heterogeneous enhancement, indicating an aggressive phenotype as well as intratumor necrosis. Pathologic examination of surgical specimens from the left posterolateral thoracotomy finally confirmed the diagnosis of MSFTP. The resection margins were tumor negative. Tissue pathologic examination revealed spindle cells separated by fibrous stroma. Immunohistochemical analysis showed that the tumor was strongly positive for CD34/CD99 and BCL-2 (B-cell lymphoma 2), weakly positive for Ki-67, and negative for S100, CKp, and CK5/6 (Figure 3); the crowded cells showed enlarged nucleus and pleomorphism, suggesting the malignancy. This immunohistochemical pattern is consistent with a diagnosis of malignant SFT. ${ }^{9}$

\section{NGS revealed a panel of altered genes}

Next, gene mutational profiling was performed using NGS, followed by Sanger sequencing validation. Our sequencing results revealed 22 alterations of 19 genes, including anaplastic lymphoma receptor tyrosine kinase (ALK), alpha thalassemia/mental retardation syndrome X-linked (ATRX), epidermal growth factor receptor (EGFR), interleukin seven receptor (IL7R), paired box 5 (PAX5), spectrin repeat containing nuclear envelope protein 1 (SYNE1), tumor protein p53 (TP53), lysine acetyltransferase 6B (KAT6B), ret proto-oncogene (RET), mutS homolog 6 (MSH6), kinase insert domain receptor (KDR), menin 1 (MEN1), fms-related tyrosine kinase 1 (FLT1), RET, nuclear receptor coactivator 2 (NCOA2), protein tyrosine phosphatase, receptor type D (PTPRD), SET domain containing 2 (SETD2), myeloproliferative leukemia virus oncogene, and phosphoinositide-3kinase regulatory subunit 1 . These findings are summarized in Table 2. Interestingly, all of these mutations occurred with a frequency ranging from $100 \%$ to $43.3 \%$, results verified by Sanger sequencing.

\section{Public data on COSMIC were used to deepen our knowledge of the altered gene panel}

To explore the biologic significance of these mutations, we referred our sequencing data to the ClinVar and COSMIC databases (Table 1), where the clinical significance and called variants gave us a preliminary idea of the pathologic effect of the mutations. However, a number of mutations demonstrated ambiguous significance or conflicting result between studies; therefore, we turned to mutation prediction databases, including PolyPhen-2 and FATHMM on COSMIC, to evaluate the potential pathogenic influence. Based on the quantified scores on both databases, FLT1-R593W, KDR-V297I, and NCOA2-R982 were estimated as pathogenic by both of the databases, while their sequencing frequencies were all around 50\%; ALK, RET, and PTPRD as well as IL7R were mutated genes that also achieved high scores in the FATHMM and PolyPhen-2 databases, respectively. However, the oncogenicities of RET and PTPRD were attenuated by the fact that their frequency was $<90 \%$ and their scores much lower in the alternative databases. To visualize the pathogenic scores by FATHMM and Polyphen-2 database, we ranked 

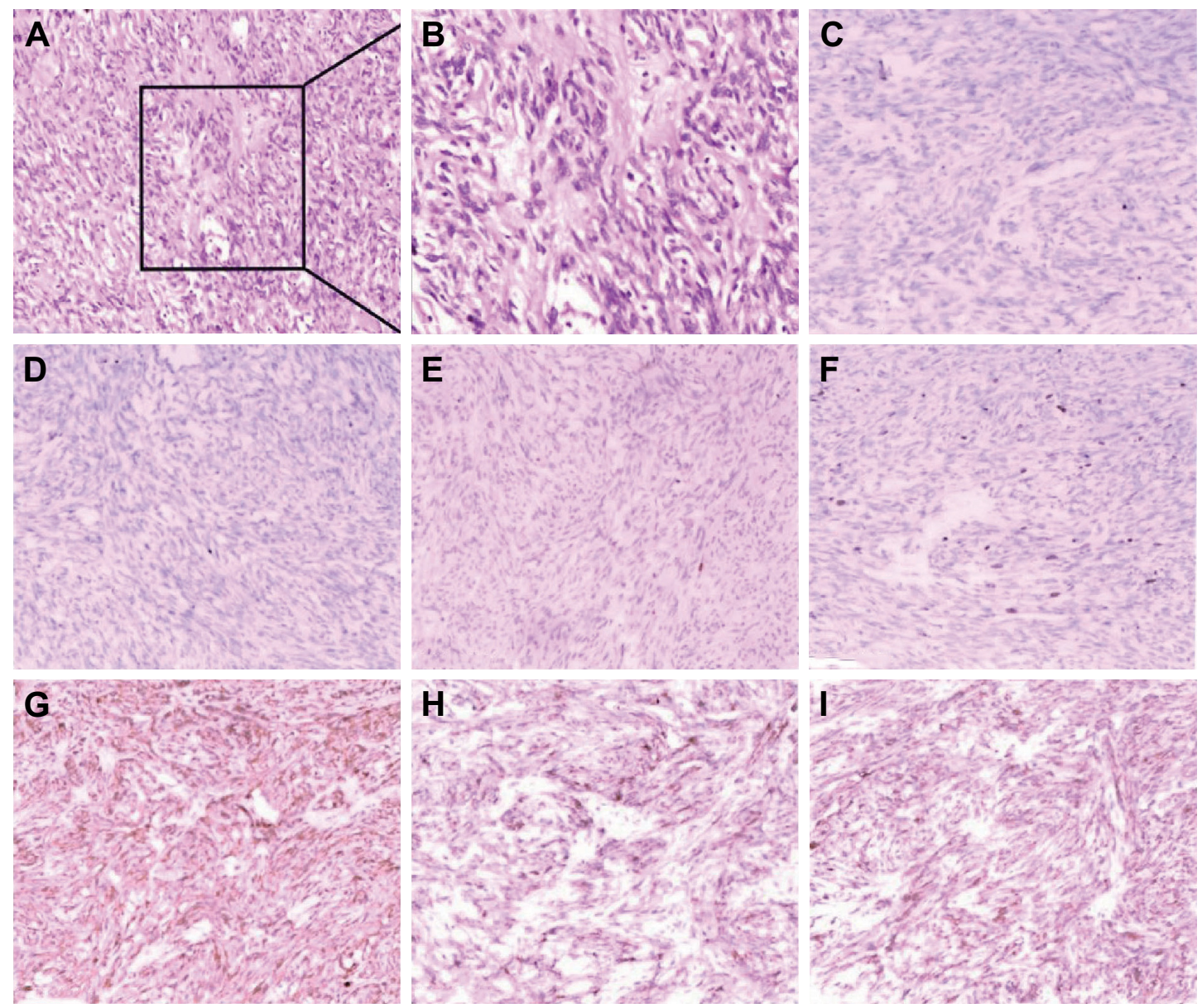

Figure 3 Immunohistochemistry staining results.

Notes: Tumor is positive for CD34, CD99, and Bcl-2, specific markers of solitary fibrous tumor of the pleura, and the positiveness of Ki-67, nucleus enlargement, and heterogeneity indicate the malignancy. (A) H\&E, $\times 100$; (B) H\&E, $\times 200$; (C) CK5/6, $\times 200$; (D) CKp, $\times 200$; (E) SI 00, $\times 200 ;$ (F) Ki-67, $\times 200$; (G) CD34, $\times 200$; (H) CD99, $\times 200$; (I) $\mathrm{Bcl}-2, \times 200$.

the mutations from Table 2 in Figure 4A. Genes that had a comparatively high mutation frequency were $A L K, A T R X$, EGFR, PAX5, SYNE1, TP53, and IL7R, but only ALK and IL7R were predicted as damaging mutations based on the two databases (Figure 4B).

We then searched for all studies of SFT on COSMIC and summarized the frequencies of all reported mutations and chromosomal alterations. Using the term "fibrous tumor", 15 studies were found. The studies conducted by Mizukami et al or Bahrami et al were excluded on the basis of irrelevance or ambiguous amino acid mutation information. In all 147 samples, NAB2-STAT6 fusions were found 125 times, representing a dominant genetic alteration of SFT. Among all gene mutations reported more than 30 times, neuroblastoma RAS viral oncogene homolog (NRAS), BRAF, platelet-derived growth factor receptor alpha, telomerase reverse transcriptase (TERT), zinc finger protein 711, and TP53 were found 9, 2, 2, 2, 2, and 2 times, respectively (Figure 4C). This method could serve to illustrate the genetic alteration "landscape" of a specific neoplasm, which is intended to be used as a characteristic of that tumor type.

Finally, our study resulted in two sets of SFT-related genetic alterations: one derived from a direct sequencing analysis of a patient with MSFTP, and the other based on further mining of publicly available datasets. Following a comparison of the mutational profiles of the two groups, we found a common genetic feature of SFT. Specifically, overlapping analysis revealed a frequently mutated gene, TP53, found altered both in our case and the COSMIC database.

\section{Discussion}

\section{Clinical characteristics}

SFTP is a primary mesenchymal neoplasm characterized by tremendous heterogeneity among studied populations. ${ }^{10}$ More specifically, SFTP refers to solitary fibrous tumors originating from the pleura, and it could be located in nearly 


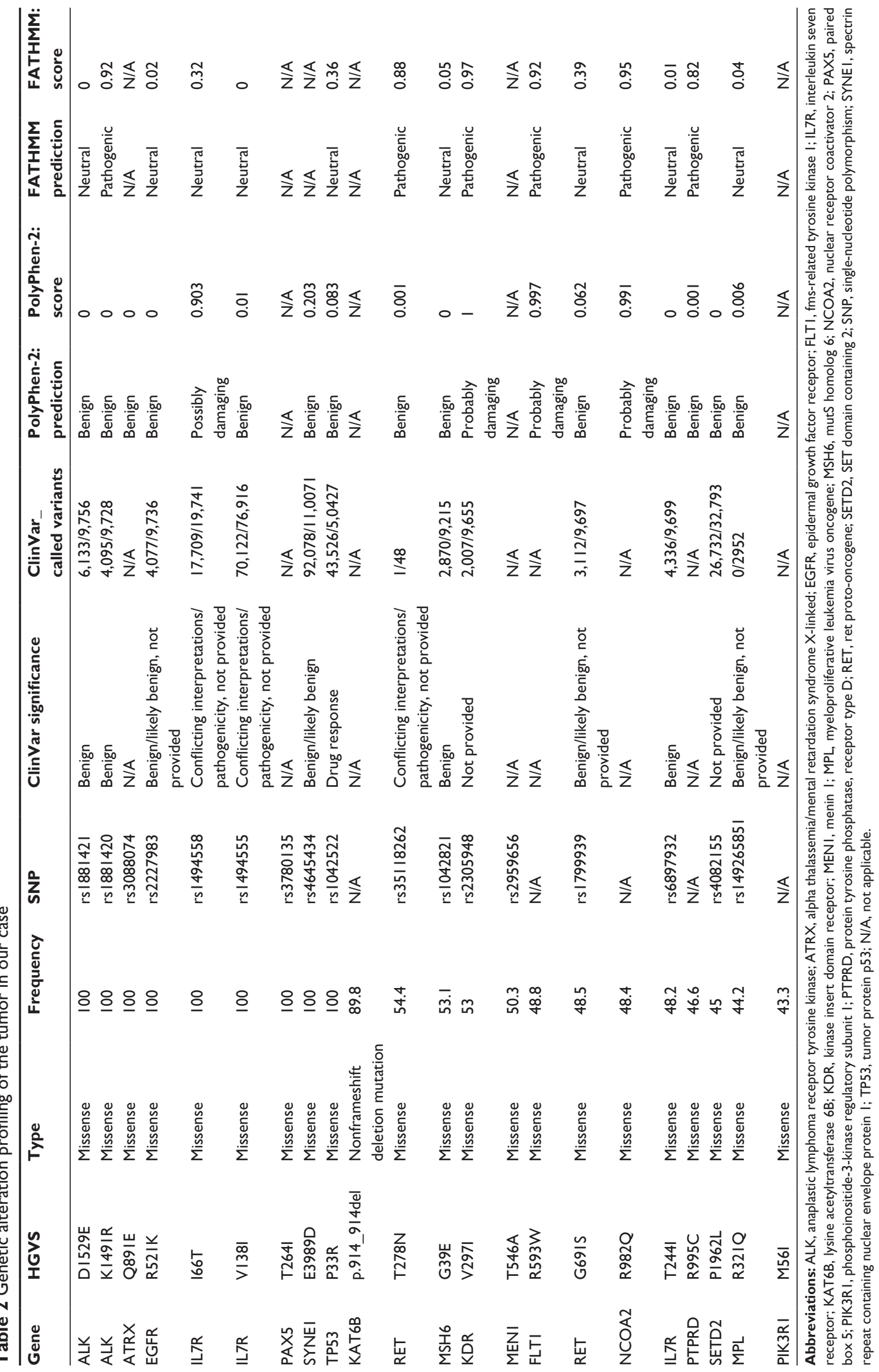


A

— FATHMM prediction: score $\quad$ PolyPhen-2: score

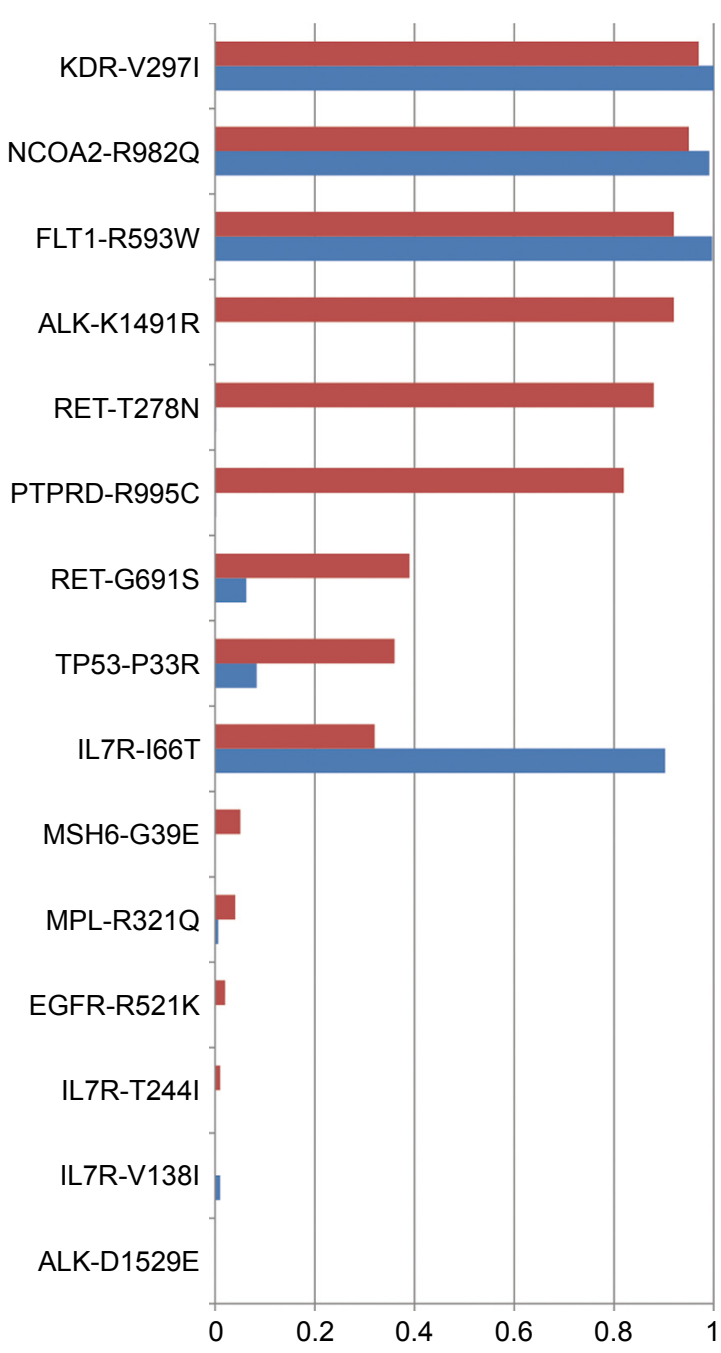

B

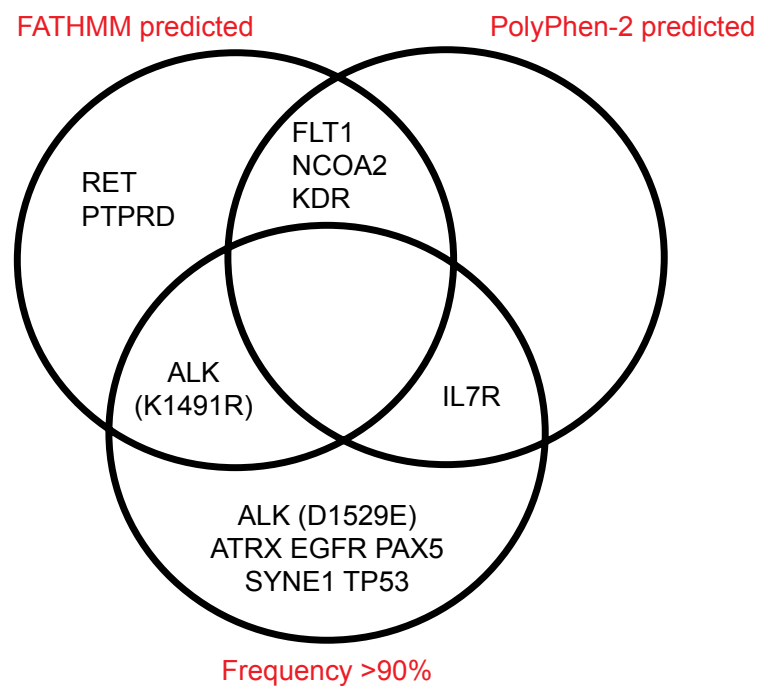

C

Pie chart of mutations and fusion for solitary fibrous tumor

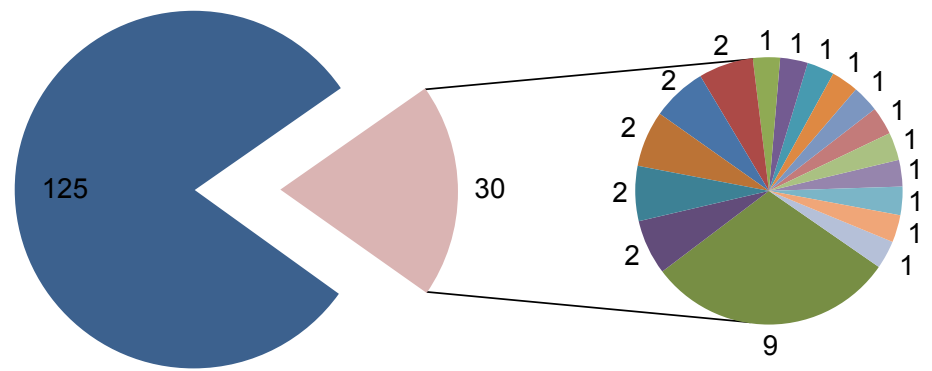

\begin{tabular}{|lll|}
\hline NAB2-STAT6 fusion & NRAS & BRAF \\
PDGFRA & $\square$ TERT & TP53 \\
ZNF711 & ARHGEF5 & ASCC3 \\
CLCA1 & EPRS & FCGR3A \\
OR2G2 & OR4K5 & RBM10 \\
RNF103 & SLFN14 & STX6 \\
\hline
\end{tabular}

Figure 4 Genetic alteration analysis and pathogenic score data.

Notes: (A) Mutations confirmed by Sanger sequencing were referred to PolyPhen-2 database (blue) and COSMIC database (red) for quantified pathogenesis score, and in this horizon line chart, mutations were ranked by FATHMM scores. (B) Pie chart of published genetic alterations of solitary fibrous tumors. (C) Mutated genes were categorized based on whether they scored high in FATHMM or COSMIC database and/or had a mutation frequency $>90 \%$, so as to hierarchically analyze the importance of the mutated genes in malignant solitary fibrous tumor of the pleura.

any organ that consists of mesothelial tissue. SFT could occur in any stage of life, with the average age of onset ranging from 51 to 66 years. ${ }^{11-13}$ While more than half of SFTPs are asymptomatic and discovered on routine chest radiography, ${ }^{10}$ large SFTPs as well as MSFTPs are usually symptomatic. ${ }^{14}$ Intrathoracic symptoms are generally nonspecific, including cough, chest pain, and dyspnea. Occasionally, patients may also report systemic symptoms such as weakness, night sweats, and weight loss. ${ }^{15}$ Extrathoracic manifestations may include the combination of arthritic pain along with clubbing of the fingers, known as Pierre MarieBamberger syndrome, ${ }^{16}$ or hypoglycemia accompanying
SFTP, referred to as Doege-Potter syndrome. ${ }^{17}$ Interestingly, paraneoplastic hypoglycemia is caused by an incompletely processed precursor - insulin-like growth factor II secreted from SFTP - and it is reversible after removal of the primary tumor. ${ }^{18-20}$

\section{Radiographic study}

$\mathrm{CT}$ and chest radiography are the primary means to detect and assess SFTP. In most cases, these imaging modalities show a well-circumscribed round/oval/lobular tumor with homogeneous density. ${ }^{21,22}$ The most common tumor location is the chest wall, with other common sites including the 
intrapulmonary/fissure space, diaphragm, and mediastinum. ${ }^{23}$ The most common SFTP shape is ovoid with a smooth margin $(n=33,80.5 \%)$, and homogeneous tumors account for the majority of SFTPs, while heterogeneity or even calcification may be observed as a result of myxoid degeneration, hemorrhage, or necrosis. ${ }^{24}$ Heterogeneity may be more apparent on contrast-enhanced CT and CT angiography reconstruction, and nourishing vessels from distant areas, a common phenomenon in malignant neoplasms, may be detected. ${ }^{25}$ In addition to heterogeneity and enhancement by the contrast medium, along with hypervascularization, tumor size above a threshold diameter of $10 \mathrm{~cm}$, presence of intratumoral hydric attenuation areas, and pleural effusion are CT features that help to distinguish malignant from benign SFTPs. ${ }^{26}$ Fludeoxyglucose ${ }^{18} \mathrm{~F}$-positron emission tomography $\left({ }^{18} \mathrm{~F}-\mathrm{FDG}-\mathrm{PET}\right)$ is broadly used for its high negative predictive value for malignancy. ${ }^{27}$ However, a clear correlation between ${ }^{18} \mathrm{~F}$-FDG-PET/CT features and malignancy of SFTPs remains under dispute, ${ }^{28}$ and a case was reported in which ${ }^{18}$ FDG-PET showed negative results for a malignant SFTP. ${ }^{29}$ The highly diverse clinical and radiographic features indicate a highly heterogeneous biologic development process, which calls for standardized pathologic evaluation.

\section{Pathology study}

SFTP is such a heterogeneous neoplasm that more than one microscopic pattern is used in the pathologic description. The first microscopic pattern is a "pattern-less" pattern: fibrous blast-like cells and connective tissues are arranged in random patterns, and the spindle/plump oval tumor cells consist of round to oval nuclei with evenly distributed chromatin and small/inconspicuous nucleoli, and eosinophilic/amphophilic poorly defined bipolar cytoplasm. ${ }^{30}$ The second pattern is a hemanigopericytoma-like pattern: closely packed tumor cells with amphiphilic cytoplasm are arranged around open or collapsed, irregular, branching capillaries and larger vessels. ${ }^{9}$ Peritumoral inflammatory adhesions are also a frequently occurring feature. ${ }^{21}$ Malignant SFTPs show greater intra- and intertumoral heterogeneity in a tumor growth pattern, cellularity, pleomorphism, and mitoses: the tumor is composed of short spindle cells together with a variable proportion of collagenous stroma. ${ }^{31}$ Criteria for distinguishing malignant SFTPs are 1) high cellularity and mitotic activity (more than four mitotic figures per 10 high power fields); 2) pleomorphism; 3) hemorrhage; and 4) necrosis. ${ }^{9}$ Similarly, in 2002, De Perrot et al also defined the malignancy of SFTP as high cellularity with crowding and overlapping of nuclei, cellular pleomorphism, high mitotic count, necrosis, or stromal/vascular invasion. ${ }^{24}$ Immunohistochemistry biomarkers for SFTPs include BCL-2 and CD34, both regarded as the dominant biomarkers for benign and malignant SFTP. ${ }^{31,32}$ Expression of CD99 and TP53 is also occasionally observed, ${ }^{32,33}$ and the latter has an intimate correlation with SFTP malignancy. Interestingly, Luis F. Tapias et al had developed a scoring system so as to predict the recurrence of SFTP after resection, including parietal pleural origin (vs visceral/intrapulmonary origin), sessile morphology (vs pedunculated), size no smaller than $10 \mathrm{~cm}$ (vs smaller than $10 \mathrm{~cm}$ ), hypercellularity, presence of necrosis or hemorrhage, and number of mitoses (per 10 high-power field). ${ }^{34}$ It is partially overlapped with the diagnosis criteria of malignancy, but it emphasized more on the predictive value, and could distinguish the comparatively aggressive SFTP cases with only pathologic features, who would relapse years later, from the totally benign and inertial cases.

\section{Molecular diagnosis and mechanisms}

NAB2-STAT6 gene fusion is another prominent and wellaccepted pathogenomic hallmark of SFTP. ${ }^{35}$ In a 52-case clinicopathologic study conducted by Huang, ${ }^{35} 51$ out of 52 cases showed distinctive STAT6 expression in the tumoral nuclei, and in 34 out of 52 cases, NAB2-STAT6 fusion was detected, a phenomenon analogously discovered by Tai in a study of 88 SFTs. ${ }^{36}$ The predominant NAB2-STAT6 type was NAB2e $\times 4$-STAT6e $\times 2 / 3$, which has been proposed to be associated with older age and pleuropulmonary tumors by Huang, ${ }^{35}$ with NAB2e $\times 6$-STAT6e $\times 16 / 17$ also exhibited. ${ }^{36}$ Once the transcription repressor NAB2 is fused with transcriptional activator STAT6, the fusion products, harboring the early growth response (EGR)-binding domain of NAB2 fused with the activation domain of STAT6, in turn oppositely activate the EGR-responsive genes and induce proliferation. ${ }^{37}$ Another RNA sequencing study of MSFTP by Cieslik revealed that oncogenic BBS9-BRAF fusion retained the kinase domain of BRAF and the truncated Ras binding domain, which may account for the aggressive phenotype of MSFTP. ${ }^{38}$ In addition, high-frequency mutations of TERT have also been detected in SFTP; strongly correlated with older age, 1 p/19q loss, and shorter DFSR; ${ }^{39,40}$ and negatively correlated with loss of ATRX expression and isocitrate dehydrogenase [NADP $(+)] 1$ 9IDH10/isocitrate dehydrogenase $[\mathrm{NADP}(+)] 1$ (IDH2) mutations. ${ }^{39}$

SFTPs are uncommon mesenchymal neoplasms with unpredictable clinical behavior and uncertain histogenesis. ${ }^{41}$ Because a single biopsy may be insufficient for identifying all gene mutations from a tumor, ${ }^{42}$ the sequencing results 
of this study were analyzed with reference to the COSMIC database to shed light on SFTP development and prognosis. As somatic mutations may serve as a genomic landscape to characterize neoplasms, we analyzed our data in two steps: we first examined the confirmed mutations in the COSMIC database for their associated neoplasms, and then evaluated the involvement of the mutated genes in SFTP. Based on the concept of multiple hits of mutation and the cardinal roles of driver mutations in tumor development, ${ }^{43-45}$ it is logical that the mutations with higher frequency may undergo mutation earlier and may have greater potential to act as the "initiating driver mutation". In the present study, ALK, ATRX, EGFR, IL7R, IL7R, PAX5, SYNE1, and TP53 showed a frequency greater than $90 \%$; however, when considering the low scores estimated by PolyPhen-2 and FATHMM for pathogenic involvement, it is difficult to say that these mutations play an initiating role in SFTP development. Interestingly, three genes with high pathogenic scores estimated by both databases, including KDR, FLT1, and NCOA2, are all receptors, and FLT1 and KDR are key receptors in angiogenesis. Given that these receptors have a comparatively medium mutation frequency and that the MSFTP lesion consists of rich nourishing vessels, we could generate the hypothesis that mutation of the VEGF receptors worked as the "second hit" that conferred upon the benign SFTP a rich angiogenic phenotype and led to the progression of malignancy.

We also collected the reported genetic alteration data for SFT from the COSMIC database, anticipating that there would be some common characteristics between our case and the public datasets. The common feature of SFTP was observed in our study: our analysis confirmed NAB2-STAT6 fusion as the major alteration in SFTP. ${ }^{36,37,40,46}$ However, we were more interested in the possible presence of genetic mutations that could serve as a characteristic landscape to predict the development and progression of SFTP. As shown in the pie chart in Figure 4C, alterations in some well-known tumor suppressor genes and oncogenes were detected in our patient, including NRAS, BRAF, and TERT. TP53 was the only overlapping gene that was found to be mutated both in our case and in other SFT cases. Although TP53 plays an indispensable role in nearly every aspect of carcinogenesis, ${ }^{47}$ we could not deem TP53 mutations as driver mutations of SFTP because the TP53-P33R mutation appears to be benign according to the PolyPhen-2 and FATHMM databases. Instead, we believe that chromosome alterations other than mutation, for example NAB2-STAT6 fusion, might play a fundamental role in this case of MSFTP. Given the diverse clinical manifestations of
SFTP, a more sophisticated pathologic classification system may aid in classification of the subtypes and enhance precision diagnosis of SFTP.

\section{Treatment and prognosis}

Patients with malignant tumors or tumors invading adjacent structures should undergo en bloc resection with wide margins of adjacent tissue. ${ }^{48}$ Negative resection margins are an important factor influencing local recurrence and metastasis. Local and general recurrences are mainly responsible for MSFTP's poor 5-year survival rate of $68 \%$. Recurrence may occur as late as 17 years after surgery, and intrathoracic recurrence can be fatal owing to mediastinal compression and vena cava obstruction resulting from extensive intrathoracic growth. ${ }^{49}$ MSFTP may also metastasize, usually by the hematogenous route, to sites including the liver, central nervous system, spleen, peritoneum, adrenal gland, gastrointestinal tract, kidney, and bone. ${ }^{50}$ Thus, local MSFTP recurrence may be impeded provided that $\mathrm{R} 0$ resection is feasible. ${ }^{51}$

Close follow-up after resection for MSFTP is crucial to ensure early detection of recurrence. Despite the high local recurrence rate after resection, the benefit of adjuvant therapy remains controversial. To date, there have been no data suggesting the potential benefit of adjuvant radiotherapy or chemotherapy after resection for MSFTP. Moreover, MSFTP has a high tumor-related mortality, and most patients who experience recurrence die as a result of the cancer within 2 years. ${ }^{48}$ Currently, a multimodal therapeutic approach is not considered mandatory in patients with MSFTP. Radiation therapy is used routinely in these patients if resection is not feasible or is incomplete. ${ }^{51}$

\section{Conclusion}

On the one hand, complete en bloc resection of SFTP provides an excellent long-term survival with low postoperative morbidity, and, therefore, provides a basal guarantee for patient prognosis. ${ }^{52}$ Recurrence is the main factor associated with poor survival after resection for MSFTP. In such cases, wide and radical resection, together with wide tumor-free margins, are necessary to minimize local recurrence and offer the best chance for cure.

On the other hand, genetic studies that explain the development of SFTP may provide insight into new remedies for prolongation of survival. Our case highlights the molecular and genetic alterations based on sequencing analysis of both this case and public datasets. A great number of cases had confirmed the high frequency of NAB2-STAT6 fusion in SFTP, while 
the somatic mutations appear to be more diverse. Although the mutational profile of our case shared little similarity with previously published data, the specific case of MSFTP presented in our study displayed a rich angiogenic phenotype that could be explained by the mutations in KDR and FLT1. As for the large gap between the mutation panel of this case and the published cases, we recommend more sophisticated classification of SFTP to reveal molecular characteristics of this complex type of tumor heterogeneity among individuals. Only with this approach could we avoid the misleading heterogeneous nonsense alterations, describe the outlines of the SFTP genetic landscape, and narrow down potential common dominant mutations or recombinations that could function as novel targets for precise diagnosis and personalized targeted medicine.

\section{Acknowledgments}

The authors gratefully thank the patient for allowing us to report this case, and are grateful to Wenhong Shen, Assistant Professor of Weill Cornell Medicine, for revision of the manuscript. This work was supported by grants from Tianjin Municipal Science and Technology Commission Fund [14JCYBJC28400], Tianjin Municipal Education Commission Fund [20120127], Tianjin Municipal Health Burea Fund [2011KZ106], and Major S\&T Special Projects of Gansu Province [143FKDH002].

\section{Author contributions}

All authors contributed toward data analysis, drafting and revising the paper and agree to be accountable for all aspects of the work. All authors reviewed and approved the final manuscript.

\section{Disclosure}

The authors report no conflicts of interest in this work.

\section{References}

1. Thway K, Ng W, Noujaim J, Jones RL, Fisher C. The current status of solitary fibrous tumor: diagnostic features, variants, and genetics. Int $J$ Surg Pathol. 2016;24(4):281-292.

2. Wagner E. Das tuberkelähnliche Lymphadenom: der cytogene oder reticulirte Tuberkel. Arch Heilk. 1870;11:495-525.

3. Bothe W, Goebel H, Kunze M, Beyersdorf F. Right atrial solitary fibrous tumor - a new cardiac neoplasm? Interact Cardiovasc Thorac Surg. 2005; 4(5):396-397.

4. Taguchi S. Primary cardiac solitary fibrous tumors. Ann Thorac Cardiovasc Surg. 2015;21(4):329-331.

5. Geramizadeh B, Marzban M, Churg A. Role of immunohistochemistry in the diagnosis of solitary fibrous tumor, a review. Iran J Pathol. 2016; 11(3): 195-203.

6. Munoz E, Prat A, Adamo B, Peralta S, Ramon y Cajal S, Valverde C. A rare case of malignant solitary fibrous tumor of the spinal cord. Spine. 2008;33(12):E397-E399.
7. Shaker W, Meatchi T, Dusser D, Riquet M. An unusual presentation of solitary fibrous tumour of the pleura: right atrium and inferior vena cava compression. Eur J Cardiothorac Surg. 2002;22(4):640-642.

8. Papadopoulos A, Porfyridis I, Christodoulides G, Georgiou A. A rare clinical case - solitary fibrous tumor of the pleura. Respir Med Case Rep. 2015;16:117-119.

9. England DM, Hochholzer L, Mccarthy MJ. Localized benign and malignant fibrous tumors of the pleura. A clinicopathologic review of 223 cases. Am J Surg Pathol. 1989;13(8):640-658.

10. Boddaert G, Guiraudet P, Grand B, Venissac N, Le Pimpec-Barthes F, Mouroux J, Riquet M. Solitary fibrous tumors of the pleura: a poorly defined malignancy profile. Ann Thorac Surg. 2015;99(3):1025-1031.

11. Soriano-Hernández MI, Husein-Elahmed H, Ruíz-Molina I. Solitary fibrous hemangiopericytoma of atypical location: importance of immunohistochemical study. Cir Cir. 2014;82(3):323-327.

12. Bishop AJ,Zagars GK, DemiccoEG, Wang WL, Feig BW, GuadagnoloBA. Soft tissue solitary fibrous tumor: combined surgery and radiation therapy results in excellent local control. Am J Clin Oncol. 2018;41(1):81-85.

13. Lococo F, Cesario A, Cardillo G, et al. Malignant solitary fibrous tumors of the pleura: retrospective review of a multicenter series. $J$ Thorac Oncol. 2012;7(11):1698-1706.

14. Sung SH, Chang JW, Kim J, Lee KS, Han J, Park SI. Solitary fibrous tumors of the pleura: surgical outcome and clinical course. Ann Thorac Surg. 2005;79(1):303-307.

15. Cardillo G, Carbone L, Carleo F, Masala N, Graziano P, Bray A, Martelli M. Solitary fibrous tumors of the pleura: an analysis of 110 patients treated in a single institution. Ann Thorac Surg. 2009;88(5):1632-1637.

16. Abu Arab W. Solitary fibrous tumours of the pleura. Eur J Cardiothorac Surg. 2012;41(3):587-597.

17. Meng W, Zhu HH, Li H, Wang G, Wei D, Feng X. Solitary fibrous tumors of the pleura with Doege-Potter syndrome: a case report and three-decade review of the literature. BMC Res Notes. 2014;7:515.

18. Kishi K, Homma S, Tanimura S, Matsushita H, Nakata K. Hypoglycemia induced by secretion of high molecular weight insulin-like growth factor-II from a malignant solitary fibrous tumor of the pleura. Intern Med. 2001;40(4):341-344.

19. Reuvers JR, van Dorp M, van Schil PE. Solitary fibrous tumor of the pleura with associated Doege-Potter syndrome. Acta Chir Belg. 2016;116(6):386-387.

20. Chang JC, Su KY, Chao SF, Hsu YH, Yang GG, Chang BS. Hypoglycemia in a patient with a huge malignant solitary fibrous tumor of the pleura. Pathol Int. 2007;57(12):791-793.

21. Magdeleinat $P$, Alifano M, Petino A, et al. Solitary fibrous tumors of the pleura: clinical characteristics, surgical treatment and outcome. Eur J Cardiothorac Surg. 2002;21(6):1087-1093.

22. Cieślik-Wolski B, Pryt Ł, Szlachcińska A, Walczak-Pasz G, JesionekKupnicka D, Kozak J. Solitary fibrous tumor of the pleura - analysis of 18 cases. Kardiochir Torakochirurgia Pol. 2015;12(3):208-215.

23. Yeom YK, Kim MY, Lee HJ, Kim SS. Solitary fibrous tumors of the pleura of the thorax: CT and FDG PET characteristics in a tertiary referral center. Medicine. 2015;94(38):e1548.

24. de Perrot M, Fischer S, Bründler MA, Sekine Y, Keshavjee S. Solitary fibrous tumors of the pleura. Ann Thorac Surg. 2002;74(1):285-293.

25. Fan F, Zhou H, Zeng Q, Liu Y. Computed tomography manifestations of a malignant solitary fibrous tumour of the pleura with distinct blood supply from celiac trunk. Eur J Cardiothorac Surg. 2014;45(6):1108-1110.

26. Hélage S, Revel MP, Chabi ML, et al. Solitary fibrous tumor of the pleura: can computed tomography features help predict malignancy? A series of 56 patients with histopathological correlates. Diagn Interv Imaging. 2016;97(3):347-353.

27. Cortes J, Rodriguez J, Garcia-Velloso MJ, Rodriguez-Espiteri N, Boan JF, Castellano JM, Torre W. [18F]-FDG PET and localized fibrous mesothelioma. Lung. 2003;181(1):49-54. 
28. Lococo F, Cafarotti S, Treglia G. Is 18 F-FDG-PET/CT really able to differentiate between malignant and benign solitary fibrous tumor of the pleura? Clin Imaging. 2013;37(5):976-977.

29. Lococo F, Rapicetta C, Ricchetti T, et al. Diagnostic pitfalls in the preoperative 18F-FDG PET/CT evaluation of a case of giant malignant solitary fibrous tumor of the pleura. Rev Esp Med Nucl Imagen Mol. 2014;33(2):109-111.

30. Stout AP. Tumors of the pleura. Harlem Hosp Bull. 1952;5(2): 54-57.

31. Zhang H, Lucas DR, Pass HI, Che M. Disseminated malignant solitary fibrous tumor of the pleura. Pathol Int. 2004;54(2):111-115.

32. Liu CC, Wang HW, Li FY, et al. Solitary fibrous tumors of the pleura: clinicopathological characteristics, immunohistochemical profiles, and surgical outcomes with long-term follow-up. Thorac Cardiovasc Surg. 2008;56(5):291-297.

33. Hiraoka K, Morikawa T, Ohbuchi T, Katoh H. Solitary fibrous tumors of the pleura: clinicopathological and immunohistochemical examination. Interact Cardiovasc Thorac Surg. 2003;2(1):61-64.

34. Tapias LF, Mercier O, Ghigna MR, et al. Validation of a scoring system to predict recurrence of resected solitary fibrous tumors of the pleura. Chest. 2015;147(1):216-223.

35. Huang SC, Li CF, Kao YC, et al. The clinicopathological significance of NAB2-STAT6 gene fusions in 52 cases of intrathoracic solitary fibrous tumors. Cancer Med. 2016;5(2):159-168.

36. Tai HC, Chuang IC, Chen TC, et al. NAB2-STAT6 fusion types account for clinicopathological variations in solitary fibrous tumors. Mod Pathol. 2015;28(10):1324-1335.

37. Robinson DR, Wu YM, Kalyana-Sundaram S, et al. Identification of recurrent NAB2-STAT6 gene fusions in solitary fibrous tumor by integrative sequencing. Nat Genet. 2013;45(2):180-185.

38. Cieslik M, Chugh R, Wu YM, et al. The use of exome capture RNA-seq for highly degraded RNA with application to clinical cancer sequencing. Genome Res. 2015;25(9):1372-1381.

39. Koelsche C, Sahm F, Capper D, et al. Distribution of TERT promoter mutations in pediatric and adult tumors of the nervous system. Acta Neuropathol. 2013;126(6):907-915.

40. Akaike K, Kurisaki-Arakawa A, Hara K, et al. Distinct clinicopathological features of NAB2-STAT6 fusion gene variants in solitary fibrous tumor with emphasis on the acquisition of highly malignant potential. Hum Pathol. 2015;46(3):347-356.
41. Schirosi L, Lantuejoul S, Cavazza A, et al. Pleuro-pulmonary solitary fibrous tumors: a clinicopathologic, immunohistochemical, and molecular study of 88 cases confirming the prognostic value of de Perrot staging system and p53 expression, and evaluating the role of c-kit, BRAF, PDGFRs (alpha/beta), c-met, and EGFR. Am J Surg Pathol. 2008;32(11): 1627-1642.

42. Zhang J, Fujimoto J, Zhang J, et al. Intratumor heterogeneity in localized lung adenocarcinomas delineated by multiregion sequencing. Science. 2014;346(6206):256-259.

43. van Gisbergen MW, Voets AM, Starmans MH, et al. How do changes in the mtDNA and mitochondrial dysfunction influence cancer and cancer therapy? Challenges, opportunities and models. Mutat Res Rev Mutat Res. 2015;764:16-30.

44. Minucci S, Monestiroli S, Giavara S, et al. PML-RAR induces promyelocytic leukemias with high efficiency following retroviral gene transfer into purified murine hematopoietic progenitors. Blood. 2002; 100(8):2989-2995.

45. Iwaya T, Mimori K, Wakabayashi G. Current topics in mutations in the cancer genome. Nihon Geka Gakkai Zasshi. 2012;113(2):185-190.

46. Kikuchi A, Fujita T, Takahashi Y, et al. Two cases of primary intracranial solitary fibrous tumor: genetic examination of NAB2-STAT6 fusion and its association with hemangiopericytoma. No Shinkei Geka. 2015; 43(7):641-648

47. Rufini A, Tucci P, Celardo I, Melino G. Senescence and aging: the critical roles of p53. Oncogene. 2013;32(43):5129-5143.

48. Imai K, Hirayama K, Matsuzaki I, et al. Resection of a giant, invasive malignant solitary fibrous tumor of pleura. Gen Thorac Cardiovasc Surg. 2012;60(12):859-862.

49. Briselli M, Mark EJ, Dickersin GR. Solitary fibrous tumors of the pleura: eight new cases and review of 360 cases in the literature. Cancer. 1981;47(11):2678-2689.

50. Flint A, Weiss SW. CD-34 and keratin expression distinguishes solitary fibrous tumor (fibrous mesothelioma) of pleura from desmoplastic mesothelioma. Hum Pathol. 1995;26(4):428-431.

51. Lahon B, Mercier O, Fadel E, et al. Solitary fibrous tumor of the pleura: outcomes of 157 complete resections in a single center. Ann Thorac Surg. 2012;94(2):394-400.

52. Supakul R, Sodhi A, Tamashiro CY, Azmi SS, Kadaria D. Solitary fibrous tumor of the pleura: a rare cause of pleural mass. Am J Case Rep. $2015 ; 16: 854-857$
OncoTargets and Therapy

\section{Publish your work in this journal}

OncoTargets and Therapy is an international, peer-reviewed, open access journal focusing on the pathological basis of all cancers, potential targets for therapy and treatment protocols employed to improve the management of cancer patients. The journal also focuses on the impact of management programs and new therapeutic agents and protocols on

\section{Dovepress}

patient perspectives such as quality of life, adherence and satisfaction The manuscript management system is completely online and includes a very quick and fair peer-review system, which is all easy to use. Visit http://www.dovepress.com/testimonials.php to read real quotes from published authors. 